Int. J. Electrochem. Sci., 14 (2019) $8781-8792$

\title{
PdAg Bimetallic Nanoparticles Encapsulated in Porous Carbon Derived from UIO-66 as Electrocatalyst for Oxygen Reduction and Hydrogen Evolution Reactions
}

\author{
Wei He ${ }^{1, *}$, Xiaoqing Li ${ }^{1}$, Zhengxin Qian ${ }^{2,3}$, Zhen Liu', and Zhenghua Tang ${ }^{3, *}$ \\ ${ }^{1}$ Chongqing Chemical Industry Vocational College, Chongqing, 401220, P. R. China \\ ${ }^{2}$ School of Pharmacy, Guangdong Pharmaceutical University, Guangzhou, 510006, P. R. China. \\ ${ }^{3}$ Guangzhou Key Laboratory for Surface Chemistry of Energy Materials, New Energy Research \\ Institute, School of Environment and Energy, South China University of Technology, Guangzhou \\ Higher Education Mega Centre, Guangzhou, 510006, China. \\ ${ }^{4}$ Department of Physics \& Engineering, Frostburg State University, Frostburg, MD 21532-2303, \\ United States. \\ *E-mail: hewei644000@163.com, zhht@ scut.edu.cn
}

doi: $10.20964 / 2019.09 .12$

Received: 2 April 2019 / Accepted: 19 June 2019 / Published: 31 July 2019

Developing dual functional electrocatalyst for both oxygen reduction reaction (ORR) and hydrogen evolution reaction (HER) is critical for promoting energy conversion efficiency in fuel cells and metalair batteries, and produce hydrogen with high purity. Herein, PdAg bimetallic nanoparticles encapsulated in porous carbon have been prepared and utilized as effective electrocatalysts for both ORR and HER. The surface microstructure, crystal structure, as well as electronic charge states of the bimetallic particles encapsulated in porous carbon were studied by transmission electron microscopy (TEM), X-ray diffraction (XRD), and X-ray photoelectron spectroscopy (XPS), respectively. In the bimetallic $\mathrm{Pd}_{\mathrm{x}} \mathrm{Ag}_{100-\mathrm{x}}$ (x represents the initial atomic Pd ratio in $\mathrm{PdAg}$ alloys) series, the $\mathrm{Pd} \mathrm{d}_{50} \mathrm{Ag}_{50}$ sample exhibited the best performance, of which its ORR activity and long-term stability were superior to $\mathrm{Pd} / \mathrm{C}$ in alkaline media along with its HER performance was comparable with $\mathrm{Pd} / \mathrm{C}$ in acid solution. The study provides a reliable method for fabricating carbon substrate supported bimetallic alloys with good activity and robust stability toward ORR, HER and beyond.

Keywords: PdAg bimetallic nanoparticles; Porous carbon; Bifunctional electrocatalyst, Oxygen reduction reaction; Hydrogen evolution reaction 
(C) 2019 The Authors. Published by ESG (www.electrochemsci.org). This article is an open access article distributed under the terms and conditions of the Creative Commons Attribution license (http://creativecommons.org/licenses/by/4.0/). 